\section{In situ Extractive Fermentation of Lactic Acid by Rhizopus oryzae in an Air-lift Bioreactor}

\author{
M. Matsumoto* and H. Furuta \\ Department of Chemical Engineering and Materials Science, \\ Doshisha University, Kyotanabe, \\ Kyoto 610-0321, Japan
}

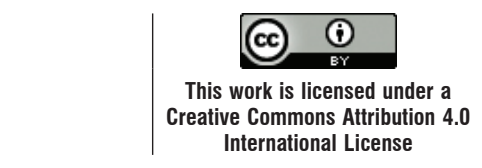

doi: 10.15255/CABEQ.2017.1208

Original scientific paper Received: September 1, 2017 Accepted: May 28, 2018

In this paper, the in situ extractive fermentation of lactic acid was developed with Rhizopus oryzae that grew in a medium without costly nutrients such as peptone or yeast extract. Tri- $n$-octylamine as an extractant and isotridecanol as a diluent were selected in an optimum extraction system from the viewpoint of the tolerance of organic solvents to $R$. oryzae and extraction capacity. Using this extraction system, batch fermentations were carried out in a stirred tank in the presence of an organic solution. It was found that a higher aeration rate was needed to enhance the production rate of the lactic acid and the extent of its extraction became relatively high due to the absence of extraction inhibitors, peptone and yeast extract. Finally, in situ extractive fermentation was carried out using pelleted $R$. oryzae in a fed-batch mode in an air-lift reactor. Lactic acid was successfully fermented and extracted from the broth to organic phase for 25 days.

Keywords:

in situ extractive fermentation, L-lactic acid, Rhizopus oryzae, trioctylamine, air-lift reactor

\section{Introduction}

Lactic acid, which plays an important role as a bulk chemical in the food, pharmaceutical, and cosmetic industries, is drawing great interest as the monomer of biodegradable poly-lactides ${ }^{1}$. Demand is increasing for optically pure lactic acid for the production of poly-lactides because its optical purity significantly affects the physical properties of poly-lactides ${ }^{2}$. Therefore, interest in the fermentative production of lactic acid is growing because the lactic acid produced by chemical synthesis routes is a racemic mixture. In addition, the biotechnological route has advantages for environmentally friendly production processes, such as low reaction temperature, low energy consumption, and the ability of using renewable resources instead of petrochemicals. The main biotechnological producers of lactic acid are bacteria and some filamentous fungi $i^{3,4}$.

The fungus Rhizopus oryzae is widely studied as an $\mathrm{L}(+)$-lactic acid producer ${ }^{3,4}$. In the fermentative production of lactic acid using fungal cells, fungi possess better resistance to high concentrations of lactic acid and low $\mathrm{pH}$ of the medium than commonly used bacteria, and use media with much lower auxotrophic levels compared to those required by bacteria ${ }^{5}$, which simplifies the downstream product separation process. Studies on the fermentative production of lactic acid have been

*To whom correspondence should be addressed.

Tel/Fax +81-774-65-6655; E-mail:mmatsumo@mail.doshisha.ac.jp conducted with pelleted and immobilized $R$. ory$z a e^{5-12}$ because free mycelia results in a highly viscous broth. The accumulation of lactic acid in the broth and the $\mathrm{pH}$ decrease in the medium associated with it negatively affect productivity ${ }^{10}$, although the fungi have better tolerance to the accumulation of lactic acid and low pH than bacteria. In situ extractive fermentation is a promising alternative to the conventional process because the accumulated lactic acid can be removed from the broth during fermentation. However, only one study ${ }^{10}$ has addressed the extractive fermentation of lactic acid with $R$. oryzae. In a previous paper ${ }^{10}$, trialkylphosphine oxide was used as the extractant of lactic acid. The reactive extraction of organic acids has already been heavily investigated ${ }^{13,14}$. Such tertiary amines like tri- $n$-octylamine (TOA), diluted in a higher alcohol like 1-octanol, effectively extracted lactic acid ${ }^{15}$. Some studies have been conducted on the in situ extractive fermentation of lactic acid with bacteria and tertiary amines ${ }^{14,16}$. One problem is the high toxicity of the extractant to bacteria. In our previous paper ${ }^{16}$, an in situ extractive fermentation process of lactic acid with TOA was constructed by co-immobilizing decanol-tolerant Lactobacillus fructivorans NRIC0224 cells and calcium carbonate into Ca-alginate capsules without $\mathrm{pH}$ control. The toxicity was considerably alleviated, but a slightly negative effect remained. As medium components, peptone and yeast extracts were found to be inhibitory compounds in the reactive extraction with TOA. 
This paper developed the in situ extractive fermentation of lactic acid with $R$. oryzae in the growth medium which did not contain peptone or yeast extract. Firstly, the toxicity of extractants and diluents to $R$. oryzae was examined. Although some studies can be found on the solvent toxicity to lactic acid producing bacteria ${ }^{17}$, our previous study is the only report that is concerned with the toxicity of diluents to lactic acid producing fungi ${ }^{18}$. The toxicity of the extractant diluted in the organic solvent was studied in this paper. In the next section, we describe our extractive fermentation experiments conducted in situ with pelleted $R$. oryzae using our selected extraction system.

\section{Materials and methods}

\section{Chemicals}

Tri- $n$-butyl phosphate (TBP), tri- $n$-hexylamine (THA), and tri-n-octylamine (TOA) were used as extractants of lactic acid. The diluents for the extractant were 1-octanol, 1-decanol, isotridecanol and oleyl alcohol. All the chemicals of GR grade having purity of $>99 \%$ were used as received from the suppliers.

\section{Organisms and cultivation}

We used a lactic acid producing mold: Rhizopus oryzae IAM 6022 . The microorganisms were maintained on potato dextrose agar (PDA, Nissui) slants. The production medium consisted of glucose $20 \mathrm{~g} \mathrm{~L}^{-1},\left(\mathrm{NH}_{4}\right)_{2} \mathrm{SO}_{4} 1.35 \mathrm{~g} \mathrm{~L}^{-1}, \mathrm{~K}_{2} \mathrm{HPO}_{4} 0.3 \mathrm{~g} \mathrm{~L}^{-1}$, $\mathrm{MgSO}_{4} \cdot 7 \mathrm{H}_{2} \mathrm{O} 0.25 \mathrm{~g} \mathrm{~L}^{-1}$ and $\mathrm{ZnSO}_{4} \cdot 7 \mathrm{H}_{2} \mathrm{O} 0.04 \mathrm{~g} \mathrm{~L}^{-16,9}$. Cultivations were performed at $30^{\circ} \mathrm{C}$.

\section{Production in the presence of organic solvents}

Lactate production in the presence of a second phase was determined based on a previous method $^{16}$. A spore suspension from a PDA slant was transferred to a $100 \mathrm{~mL}$ production medium in a $250-\mathrm{mL}$ culture bottle. The culture bottle was shaking-incubated for $24 \mathrm{~h}$ at $30{ }^{\circ} \mathrm{C}$ and $100 \mathrm{rpm}$ (BW201, Yamato, Tokyo Japan). After pH of the medium was adjusted to 6 by adding a $5 \mathrm{~mol} \mathrm{~L}^{-1}$ $\mathrm{NaOH}$ aqueous solution, we added $30 \mathrm{~mL}$ of each organic solvent listed in Table 1 to form the second phase. The culture bottles were sealed with Teflon valves to prevent evaporation and incubated for 48 $\mathrm{h}$ at $30^{\circ} \mathrm{C}$. The glucose concentration was then measured.

\section{Batch fermentation in a stirred tank}

Batch fermentations were performed with a working volume of $600 \mathrm{~mL}$ at $30^{\circ} \mathrm{C}$ in $1-\mathrm{L}$ stirred tank (MBF, EYELA, Tokyo Japan). A spore suspen-
Table 1 - Toxicity of organic solvents to Rhizopus oryzae IAM 6022

\begin{tabular}{lcc}
\hline \multicolumn{1}{c|}{ Solvent } & $\log P_{\mathrm{o} / \mathrm{w}}$ & Relative activity \\
\hline 1-Octanol & 2.9 & $\approx 0$ \\
1-Decanol & 3.8 & 0.21 \\
Isotridecanol & 5.2 & 0.70 \\
Oleyl alcohol & 7.5 & 1.09 \\
Trioctylamine (TOA) & 10.3 & 0.056 \\
Trihexylamine & 7.4 & 0.005 \\
Tributyl phosphate & 3.8 & $\approx 0$ \\
0.5 mol L & \\
0.5 mol LOA in isotridecanol & - & 0.99 \\
\hline
\end{tabular}

Relative activity is defined as ratio of glucose consumption of sample to that of control.

sion from a PDA slant was transferred to a $600 \mathrm{~mL}$ production medium. The aeration rate and agitation speed were $1.0 \mathrm{~L} \mathrm{~min}^{-1}$ and $70 \mathrm{rpm}$. In the anaerobic condition, air was not bubbled. Samples were withdrawn periodically during the fermentation, analysed for glucose and lactic acid and measured $\mathrm{pH}$ value. In the absence of an organic phase, the $\mathrm{pH}$ of the broth was manually kept at the initial $\mathrm{pH}$ by adding a $5 \mathrm{~mol} \mathrm{~L}^{-1} \mathrm{NaOH}$ solution. In the case of extractive fermentation, $200 \mathrm{~mL}$ of organic solution, which consisted of $0.5 \mathrm{~mol} \mathrm{~L}^{-1} \mathrm{TOA}$ in isotridecanol, was added to the broth. Extractive fermentation was conducted without $\mathrm{pH}$ control.

\section{Pellet formation}

A spore suspension from a PDA slant was transferred to a $100 \mathrm{~mL}$ production medium in a 250-mL culture bottle, which was shaking-incubated for $72 \mathrm{~h}$ at $28^{\circ} \mathrm{C}$ and $100 \mathrm{rpm}$ (BW201, Yamato, Tokyo Japan). During the incubation, the $\mathrm{pH}$ was kept at the initial $\mathrm{pH}$ level of the culture by adding calcium carbonate to prompt a morphology change from dispersed filamentous to a pellet form 3 to 4 $\mathrm{mm}$ in diameter. Pellet formation was confirmed visually.

\section{Extractive fermentation of lactic acid with pelleted $R$. oryzae in air-lift reactor}

We also carried out lactic acid production using pelleted $R$. oryzae in an air-lift reactor (diameter $120 \mathrm{~mm}$, length $250 \mathrm{~mm}$ ) in a batch mode as illustrated in Fig. 1. The volumes of culture including 5 $\mathrm{mL}$ of antifoaming agent (Tween 80 ) and extraction phase were initially $2 \mathrm{~L}$ and $500 \mathrm{~mL}$, respectively. The broth in the tank was held at $28{ }^{\circ} \mathrm{C}$ and the aeration rate from annular air tube was $6.0 \mathrm{~L} \mathrm{~min}^{-1}$. In extractive fermentation, $500 \mathrm{~mL}$ of isotridecanol solution containing $0.5 \mathrm{~mol} \mathrm{~L}^{-1} \mathrm{TOA}$ was placed on 


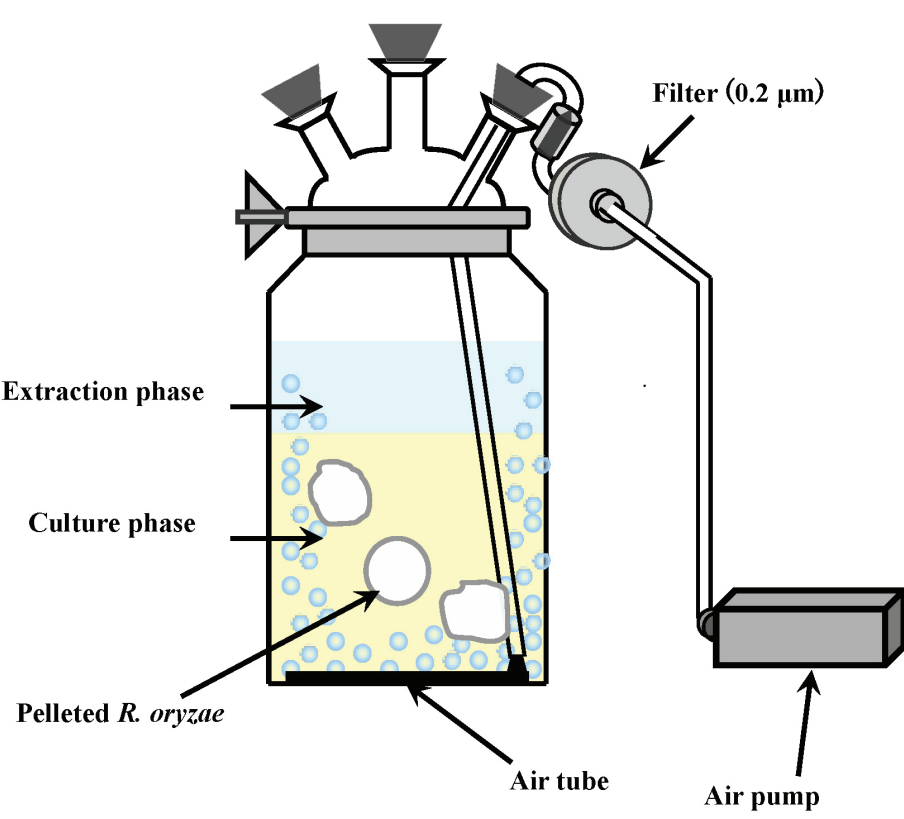

Fig. 1 - Schematic diagram of extractive fermentation with air-lift reactor

top of the medium solution. Samples were withdrawn periodically from the medium and the organic phase during fermentation, and analysed for $\mathrm{pH}$, glucose and lactic acid. When the $\mathrm{pH}$ of the broth decreased to 3 , the extraction phase was exchanged with a fresh one. When the glucose was depleted, an enriched $100 \mathrm{~mL}$ glucose solution was added to become an initial glucose concentration.

\section{Analysis}

The lactic acid in the organic phase was stripped with a $1 \mathrm{~mol} \mathrm{~L}^{-1} \mathrm{NaOH}$ solution. The concentrations of the lactic acid, glucose and ethanol in the resultant aqueous solutions were determined by HPLC (Shimadzu LC-10ADvp) with a Shodex SH1011 column and a $5 \mathrm{mmol} \mathrm{L}^{-1} \mathrm{H}_{2} \mathrm{SO}_{4}$ solution as a mobile phase. Lactic acid, glucose and ethanol were detected with an RI detector (Shimadzu RID10A).

\section{Results and discussion}

\section{Tolerance of $\boldsymbol{R}$. oryzae to organic solvent}

We previously ${ }^{18}$ investigated the tolerance of lactic acid-producing fungi $R$. oryzae JCM 5568 to organic solvents. A solvent with a high $\log P_{\mathrm{o} / \mathrm{w}}$ exhibited the highest metabolic activities and solvents with a lower $\log P_{\mathrm{o} / \mathrm{w}}\left(P_{\mathrm{o} / \mathrm{w}}\right.$ is the partition coefficient of a molecule between 1-octanol and water) were toxic to $R$. oryzae JCM 5568, which showed higher tolerance to aliphatic alcohols than to aliphatic hydrocarbons. Furthermore, long-chain aliphatic alcohols, such as octanol and decanol, were reported to be active diluents for lactic acid extraction ${ }^{19}$. We examined the tolerance of $R$. oryzae IAM 6022 to long-chain aliphatic alcohols as diluents and extractants such as TBP, THA and TOA. Table 1 shows the relationship between the relative activity retained by cells exposed to long-chain aliphatic alcohols and extractants, and their $\log P_{\mathrm{o} / \mathrm{w}}$ values. Relative activity in the presence of organic solvents was determined on the basis of the concentration of the glucose consumed after adding an organic solvent, where the relative activity was defined as the ratio of the glucose concentration of the sample to that of the control. Aliphatic alcohols with higher $\log P_{\mathrm{o} / \mathrm{w}}$ were less toxic to $R$. oryzae IAM $6022^{18}$. All the extractants used in this study showed high toxicity to $R$. oryzae IAM 6022 . Although oleyl alcohol produced the highest activity, one of its disadvantage is its relatively high viscosity of $28.32 \mathrm{mPa} \mathrm{s}$ at $25^{\circ} \mathrm{C}^{20}$. Therefore, we examined the tolerance of a mixture of TOA and isotridecanol or 1-decanol. In the case of TOA $\left(0.5 \mathrm{~mol} \mathrm{~L}^{-1}\right)$ and isotridecanol, its relative activity is comparable to that of control. In the following experiment, this extraction system was employed.

\section{Extractive fermentation in a stirred tank}

Based on the above results, batch fermentations were carried out in a stirred tank to examine the effect of the addition of an organic solution on fermentation. Figs. 2 and 3 show the time courses of the concentrations of glucose, lactic acid and ethanol, and $\mathrm{pH}$ during fermentation in the absence and presence of an organic solution, respectively. In $\mathrm{pH}$-controlled fermentation without an organic solution, the glucose was completely depleted at $68.5 \mathrm{~h}$, and lactic acid and ethanol were produced $\left(Y_{\mathrm{P} / \mathrm{S}}=\right.$ lactic acid produced/glucose consumed = 0.492 g-lactic acid $\mathrm{g}^{-1}$-glucose). For the extractive fermentation, $\mathrm{pH}$ was uncontrolled because the condition of the lower $\mathrm{pH}$ was preferable for the extraction $^{16}$. Lactic acid was successfully produced by fermentation in the presence of an organic solution. However, the fermentation rate was slower than that of the control, and the time required to deplete the glucose was extended to $120 \mathrm{~h}$, although the presence of $0.5 \mathrm{~mol} \mathrm{~L}^{-1} \mathrm{TOA}$ in the isotridecanol had little effect on the lactic acid production as described above. This suggests that air supply for $R$. oryzae was insufficient because the layered organic phase cut off the air contact. Fig. 4 shows extractive fermentation under anaerobic conditions. As is evident from Figs. 2 and 4, the anaerobic fermentation is slower than the aerobic fermentation. Therefore, a higher aeration rate is needed to enhance the fermentation rate. After 120 h, $55 \%$ of lactic acid produced was extracted to the organic phase at $\mathrm{pH} 4$. In our previous paper ${ }^{16}$, it was reported that yeast ex- 


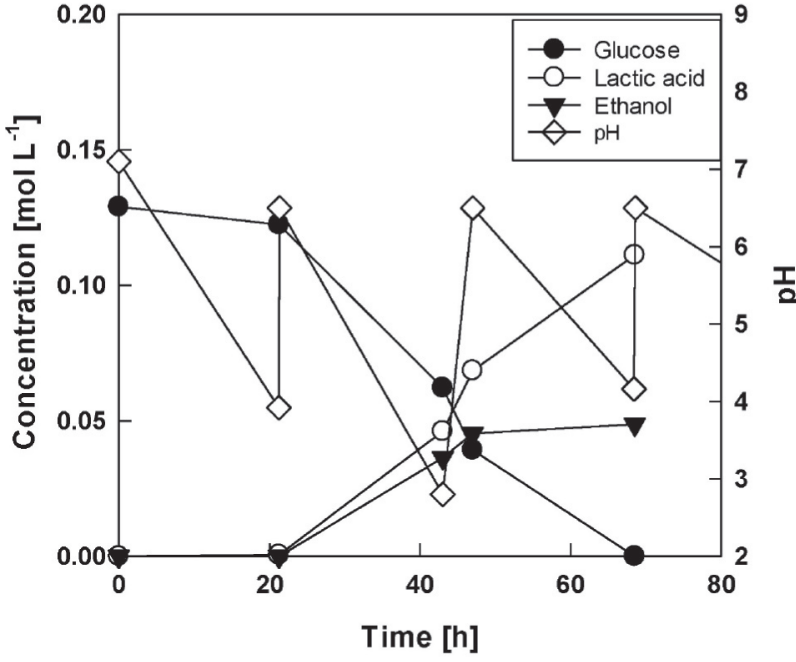

Fig. 2 - Lactic acid fermentation by Rhizopus oryzae IAM 6022 in stirred tank under aerobic conditions

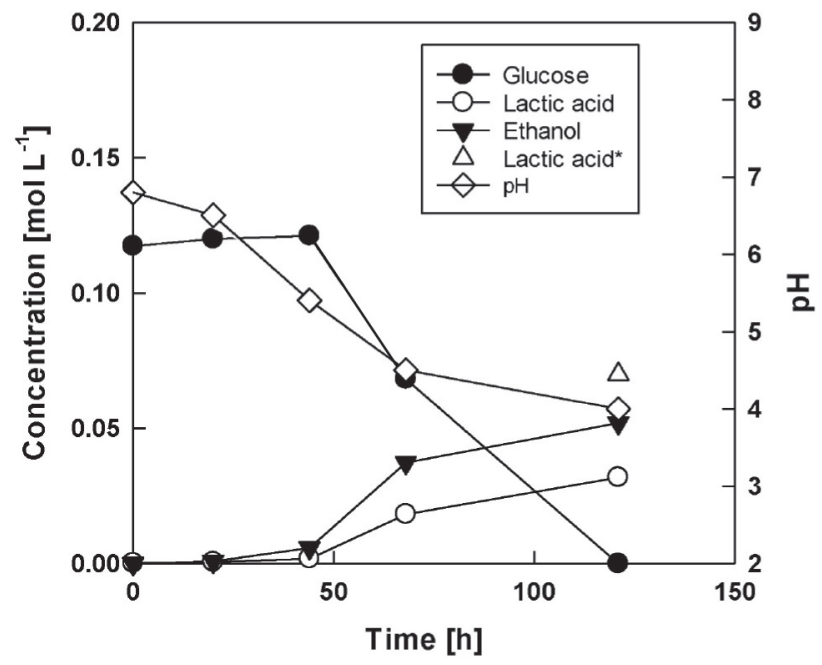

Fig. 3 - Extractive fermentation of lactic acid by Rhizopus oryzae IAM 6022 in stirred tank under aerobic conditions (*lactic acid produced considering lactic acid extracted in the organic phase)

tract and peptone in the broth considerably interfered with the extraction of lactic acid and extractability in the broth decreased to $1 / 10$ compared to that of the model solution in the absence of yeast extract and peptone. In this experiment, relatively high extractability was obtained, because the medium did not contain yeast extract or peptone.

\section{Extractive fermentation in an air-lift reactor}

The application of fungi such as Rhizopus for the commercial production of lactic acid was considered using air-lift reactors because of their low energy consumption ${ }^{4}$. As described above, a high aeration rate is needed to enhance the fermentation

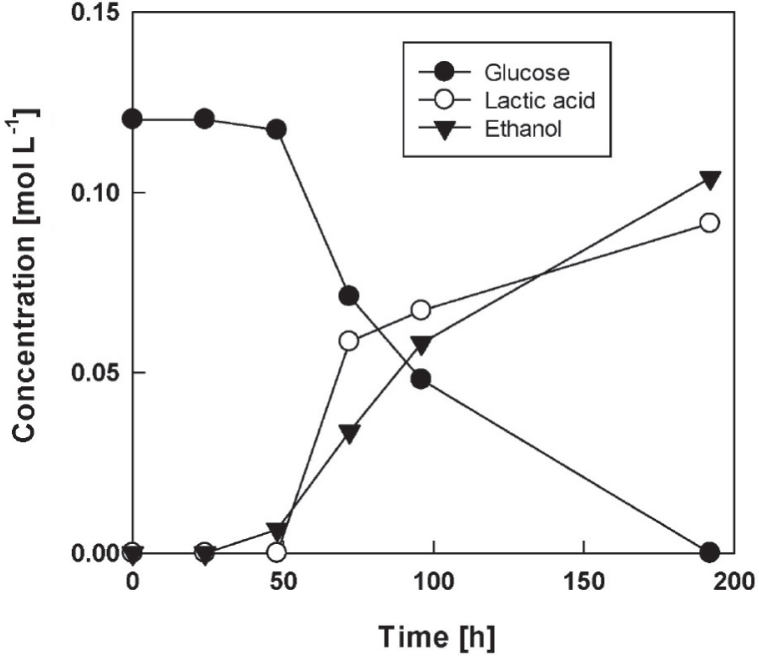

Fig. 4 - Lactic acid fermentation by Rhizopus oryzae IAM 6022 in stirred tank under anaerobic conditions

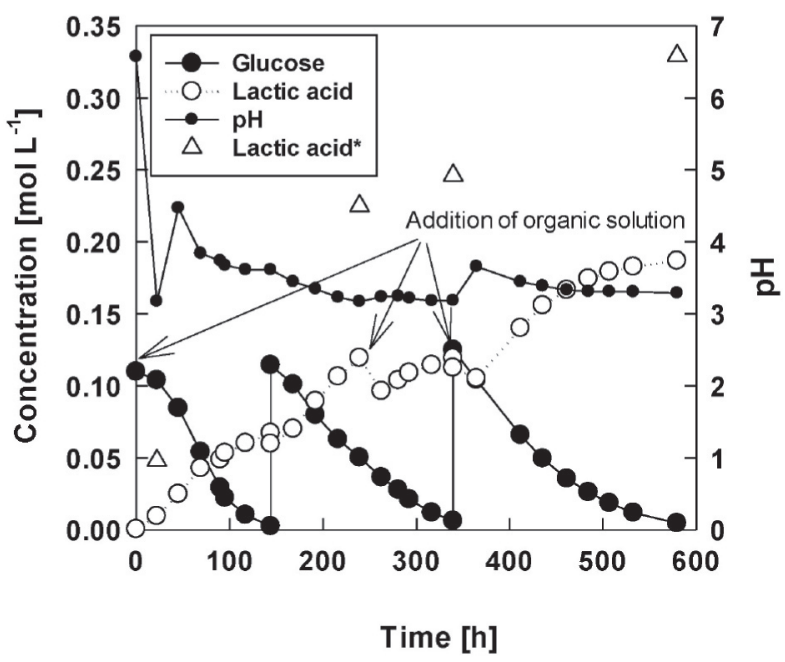

Fig. 5 - Extractive fermentation of lactic acid by Rhizopus oryzae IAM 6022 in air-lift reactor (*lactic acid produced considering lactic acid extracted in organic phase)

rate. In this study, in situ extractive fermentation was carried out using pellets of $R$. oryzae in a fedbatch mode in air-lift reactor shown in Fig. 1.

Fig. 5 shows the time courses of the glucose and lactic acid concentrations in the extractive fermentation of lactic acid in an air-lift bioreactor without $\mathrm{pH}$ control. When the glucose was depleted in the broth, $100 \mathrm{~mL}$ enriched glucose solution was added so that it became an initial glucose concentration. We continued the fermentation until the glucose was depleted in the third cycle. Yield, $Y_{\mathrm{P} / \mathrm{S}}$, glucose consumption rate, $v_{\text {glu }}$, fermentation time (FT), and efficiency of extraction, $E$, in each cycle are listed in Table 2. These constants were defined by the following equations. 


$$
\begin{gathered}
\left.Y_{\mathrm{P} / \mathrm{S}}=\text { lactic acid produced/glucose consumed } \cdot 100 \text { [g-lactic acid } \mathrm{g}^{-1} \text {-glucose }\right] \\
v_{\text {glu }}=\text { concentration change of glucose/FT }\left[\text { mmol-glucose } \mathrm{L}^{-1} \mathrm{~h}^{-1}\right] \\
\text { FT }=\text { time from glucose addition to glucose consumption }[\mathrm{h}]
\end{gathered}
$$

From Fig. 5, the lactic acid was successfully fermented during each cycle and extracted to the organic phase for $600 \mathrm{~h}$. The yield in an air-lift bioreactor exceeded that $(=98 \%)$ in an aerobic batch reactor shown in Fig. 2. In an air-lift bioreactor, the $\mathrm{pH}$ gradually decreased after the initial stage, and when it fell to 3.3, the organic phase was replaced with a fresh one, because an extraction equilibrium was achieved. As is evident from Table 2, however, after repeated cycles, the yield and glucose consumption rate slightly decreased, and the fermentation time was prolonged, probably due to the slight toxicity of the organic phase. The extractability of lactic acid from the broth remained unchanged from the model solution described in the previous paper ${ }^{16}$.

Future work will introduce a circulation system of an organic solution combined with a stripping process to continuously recover lactic acid from the fermentation broth.

\section{Conclusion}

This paper developed the in situ extractive fermentation of lactic acid with Rhizopus oryzae that grew in a medium without costly nutrients, such as peptone and yeast extract. Firstly, we examined a suitable organic solution for this fermentation system, and selected tri- $n$-octylamine as an extractant and isotridecanol as a diluent based on the tolerance of organic solvents to $R$. oryzae and extraction capacity. Using this extraction system, batch fermentations were carried out in a stirred tank to examine the effect of the addition of an organic solution on the fermentation. We found that a higher aeration rate was needed to enhance the production rate of the lactic acid and the extent of lactic acid extracted became relatively high due to the absence of extraction inhibitors such as peptone and yeast extract. Finally, in situ extractive fermentation was carried out using pellets of $R$. oryzae in a fed-batch mode in an air-lift reactor. Lactic acid was successfully fermented and extracted to an organic phase for $600 \mathrm{~h}$.

Table 2 -Constants of extractive fermentation in air-lift reactor

\begin{tabular}{c|ccccc}
\hline Cycle & $Y_{\mathrm{P} / \mathrm{S}}(\%)$ & $v_{\text {glu }}\left(\mathrm{mmol} \mathrm{L}^{-1} \mathrm{~h}^{-1}\right)$ & $\mathrm{FT}(\mathrm{h})$ & $E(\%)$ \\
\hline 1st & 66 & 7.46 & 144 & $46.9^{*}$ \\
2nd & 57 & 5.53 & 196 & 52.9 \\
3rd & 48 & 5.01 & 240 & 43.2 \\
\hline
\end{tabular}

*This value was measured at $239 \mathrm{~h}$.
Having developed optimal fungi for lactic acid production, this paper demonstrates that extractive fermentation is a promising method.

\section{ACKNOWLEDGMENTS}

This work was supported by JSPS KAKENHI Grant Number 16H04556 and the MEXT-Supported Program for the Strategic Research Foundation at Private Universities.

\section{References}

1. Nampoothiri, K. M., Nair, N. R., John, R. P., An overview of the recent developments in polylactide (PLA) research, Bioresource Technol. 101 (2010) 8493. doi: https://doi.org/10.1016/j.biortech.2010.05.092

2. Inkinen, S., Hakkarainen, Albertson, A.-C., Södergård, A., From lactic acid to poly(lactic acid) (PLA): Characterization and analysis of PLA and its precursors, Biomacromolecules 12 (2011) 523. doi: https://doi.org/10.1021/bm101302t

3. Ghaffar, T., Irshad, M., Anwar, Z., Aqil, T., Zulifqar, Z., Tariq, A., Kamran, M., Ehsan, N, Mehmood, S., Recent trends in lactic acid biotechnology: A brief review on production to purification, J. Radiation Res. Appl. Sci. 7 (2014) 222 doi: https://doi.org/10.1016/j.jrras.2014.03.002

4. Juturu, $V$., $W u, J . C$., Microbial production of lactic acid: The latest development, Crit. Rev. Biotechnol. 36 (2016) 967. doi: https://doi.org/10.3109/07388551.2015.1066305

5. Ranjit, C., Srividya, S., Lactic acid production from free and polyurethane immobilized cells of Rhizopus oryzae MTCC 8784 by direct hydrolysis of starch and agro-industrial waste, Int. Food Res. J. 23 (2016) 2646.

6. Miura, S., Arimura, T., Hoshino, M., Kojima, M., Dwiarti, L., Okabe, M., Optimization and scale-up of L-lactic acid fermentation by mutant strain Rhizopus sp. MK-96-1196 in airlift bioreactors, J. Biosci. Bioeng. 96 (2003) 65. doi: https://doi.org/10.1016/S1389-1723(03)90098-3

7. Bai, D., Jia, M., Zhao, X., Ban, R., Shen, F., Li, X., Xu, S., $\mathrm{L}(+)$-Lactic acid production by pellet-form Rhizopus oryzae R1021 in a stirred tank fermentor, Chem. Eng. Sci. 58 (2003) 785. doi: https://doi.org/10.1016/S0009-2509(02)00608-5

8. Koide, M., Hirata, M., Gao, M., Takanashi H., Hano, T., Continuous fermentation of L-lactic acid by the mold Rhizopus oryzae, Kagaku Kogaku Ronbunshu 30 (2004) 432. doi: https://doi.org/10.1252/kakoronbunshu.30.432

9. Miura, S., Dwiarti, L., Okabe, M., Enhanced production of L-lactic acid by ammonia- tolerant mutant strain Rhizopus sp. MK-96-119, J. Biosci. Bioeng. 97 (2004) 19. doi: https://doi.org/10.1016/S1389-1723(04)70159-0

10. Lin, J., Zhou, M., Zhao, X., Luo, S., Lu, Y., Extractive fermentation of L-lactic acid with immobilized Rhizopus oryzae in a three-phase fluidized bed, Chem. Eng. Process. 46 (2007) 369.

doi: https://doi.org/10.1016/j.cep.2006.06.015 
11. Wu, X., Jiang, S., Liu, M., Pan, L., Zheng, Z., Luo, S., Production of L-lactic acid by Rhizopus oryzae using semicontinuous fermentation in bioreactor, J. Ind. Microbiol. Biotechnol. 38 (2011) 565

doi: https://doi.org/10.1007/s10295-010-0804-8

12. Vodnar, D. C., Dulf, F. V., Pop, O. L., Socaciu, C., L(+)-Lactic acid production by pellet-form Rhizopus oryzae NRRL 395 on biodiesel crude glycerol, Microbial Cell Factories 12 (2013) 92.

doi: https://doi.org/10.1186/1475-2859-12-92

13. Li, Q. Z., Jiang, X. L., Feng, X. J., Wang, J. M., Sun, C., Zhang, H. B., Xian, M., Liu, H. Z., Recovery processes of organic acids from fermentation broths in the biomass-based industry, J. Microbiol. Biotechnol. 26 (2016) 1. doi: https://doi.org/10.4014/jmb.1505.05049

14. Komesu, A., de Oliveria, J. A. R., Martins, L. H. da S., Maciel, M. R. W., Filho, R. M., Lactic acid production to purification: A review, BioResources 12 (2017) 1. doi: https://doi.org/10.15376/biores.12.2.Komesu

15. Krzyzaniak, A., Leeman, M., Vossebeld, F., Visser, T. J., Schuur, S., de Haan. A. B., Novel extractants for the recovery of fermentation derived lactic acid, Sep. Purif. Technol. 111 (2013) 82.

doi: https://doi.org/10.1016/j.seppur.2013.03.031
16. Matsumoto, M., Nishimura, M., Kobayashi, H., Kondo, K., Extractive fermentation of lactic acid with Hiochi bacteria in a two-liquid phase system, Ferment. Technol. 5 (2016) 1000129.

doi: https://doi.org/10.4172/2167-7972.1000129

17. Matsumoto, M., Mochidzuki, K., Kondo, K., Toxicity of ionic liquids and organic solvents to lactic acid-producing bacteria, J. Biosci. Bioeng. 98 (2004) 344. doi: https://doi.org/10.1016/S1389-1723(04)00293-2

18. Matsumoto, M., Aoki, K., Kondo, K., Toxicity of organic solvents to Rhizopus oryzae JCM 5568, Solv. Extr. Res. Develop. Japan 15 (2008) 127.

19. Tik, N., Bayraktar, E., Mehmetoglu, Ü., In situ reactive extraction of lactic acid from fermentation media, J. Chem. Tech. Biotechnol. 76 (2001) 764. doi: https://doi.org/10.1002/jctb.449

20. Blahušiak, M., Marták, J., Miranda, F., Schlosser, S., Teixeira, J. S., Effect of viscosity of a liquid membrane containing oleyl alcohol on the pertraction of butyric acid, Chem. Paper 67 (2013) 1560. doi: https://doi.org/10.2478/s11696-013-0370-4 